\title{
P-PB-017
}

\author{
IMPLANT THERAPY \\ OUTCOMES, PERI-IMPLANT \\ BIOLOGY ASPECTS
}

\section{Immediate Molar Implant Placement and The Anatomic Harmony Abutment; a case series}

\author{
M.Gozlu ${ }^{1}$, H.Ozdemir', M.Inan²
}

${ }^{1}$ DDS PhD Private Periodontist, Dentestetik Dental Center, Konya/TR

${ }^{2}$ DDS Private Practice, Dentestetik Dental Center, Konya/TR

\section{Abstract}

Background: The placement of immediate implants in the posterior sector is a widespread procedure where the success and survival rates are similar to those of traditional protocols. However, large extraction sockets are difficult to seal without mucoperiosteal flap reflection, an implant preparation sequence that considerably deviates from typical manufacturer protocols, crestal socket morphology not amenable to standard healing abutment placement and the possibility of high occlusal forces with inadvertent function when a provisional implant is planned. Aim/Hypothesis: The purpose of this case series are to assess related technique that will allow chairside manufacturing of custom healing abutments as also called the anatomic healing abutment for immediate molar placement.
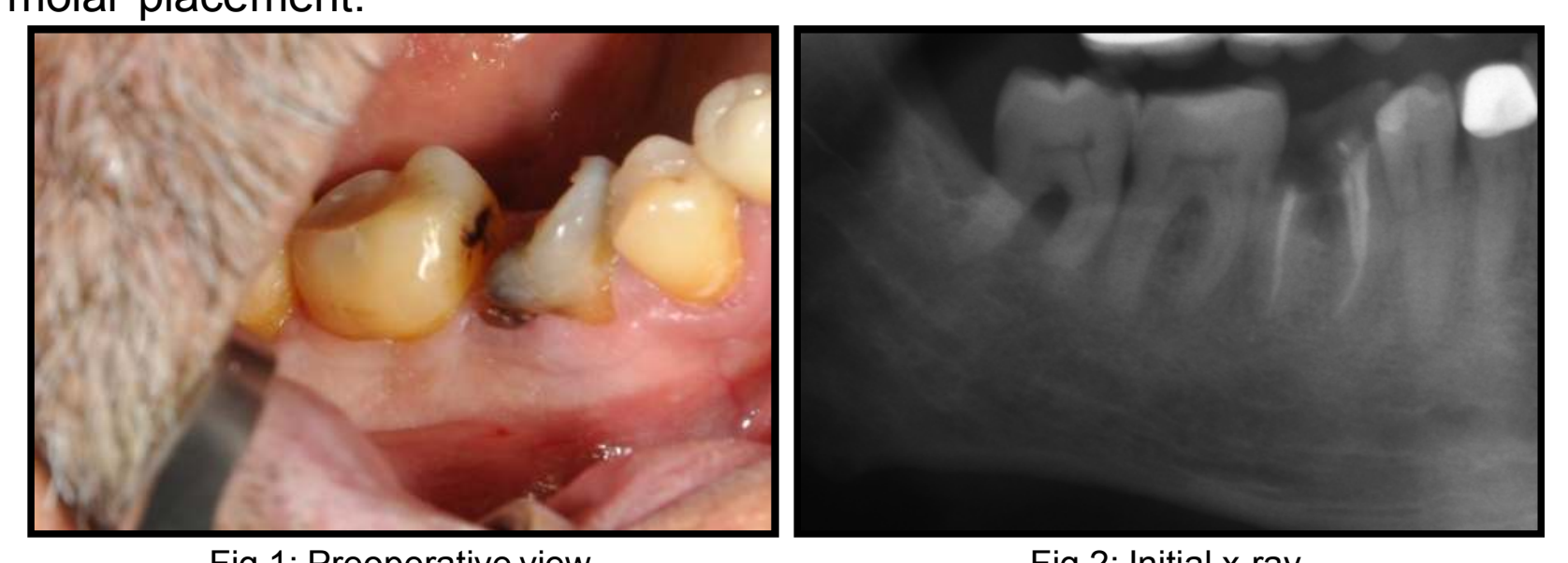

Fig 1: Preoperative view

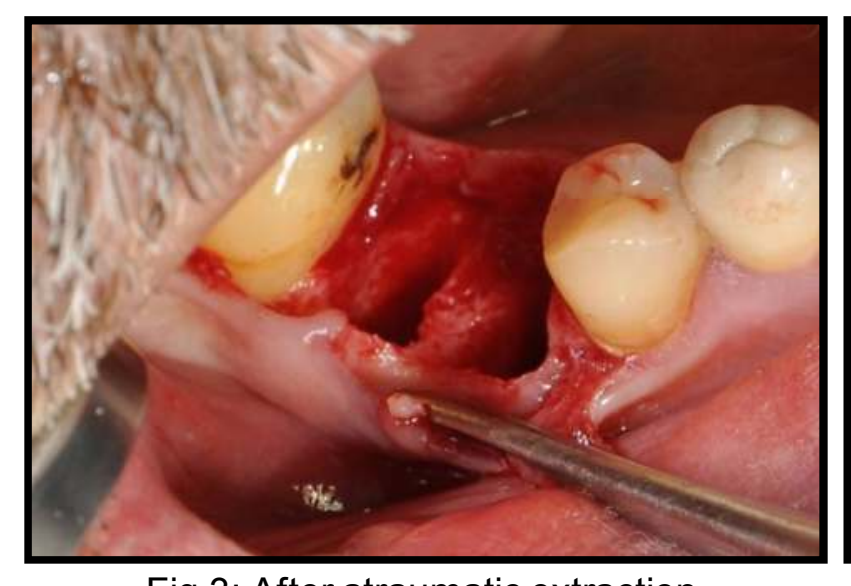

Fig 2: Initial x-ray

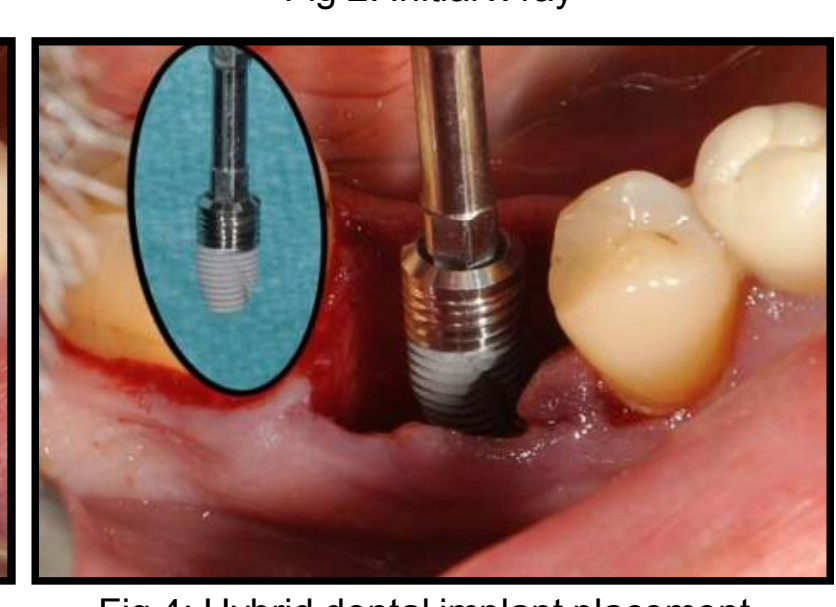

Fig 4: Hybrid dental implant placement

Fig 3: After atraumatic extraction

\section{Methods and Materials}

A 55-year-old nonsmoking male patient, with no systemic condition and a 42 year female healthy with no significant medical history patient had lost her lower first molars due to nonrestorable extensive decay. According to clinical and radiographic examination on the patient detecting decayed remaining roots in molar tooth and performed an extensive restoration presenting deep, subgingival distal decay on tooth. So that, we decided to extract related tooth and then perform immediate implant placement. After minimally traumatic tooth extractions, the alveolus was carefully cleaned and washed surgically with a saline solution, and dental implants (iRES SAGL, Switzerland) was placed in the center of the interradicular bone, in a type A position according to the Tarnow classification. After then a temporary abutment was fabricated utilizing flowable composite, which adapted to the socket architecture, to serve as a customized healing screw. It is called the anatomic or customised healing abutment. This technique preserves the anatomic emergence form with sutureless implant site sealing and improves the predictability of final restoration fabrication and delivery. After 8 weeks of the Osseo integrated period, the custom healing screw served successfully as a tooth profile for periimplant tissue.

\section{Conclusion}

Implants may be placed in ideal restorative positions at the time of mandibular molar extraction. With the anatomic harmony abutment technique, the soft tissues upon maturation closely resemble the natural root contour, which allows for the generation of esthetic and functional implantsupported restorations. 5 .

\section{Results}

Uneventful healing was observed durig this time period in this cases. It is important to note that the initial contour appeared to have been maintained. The anatomic harmony abutment ultimately allows for more predictable final restorations by providing the dental laboratory a restorative profile. thus idealizing the final restoration. The soft tissue margin was stable and the esthetic appearance of the implant site was well maintained and with no clinical signs of peri-implant inflammation and bleeding on probing.

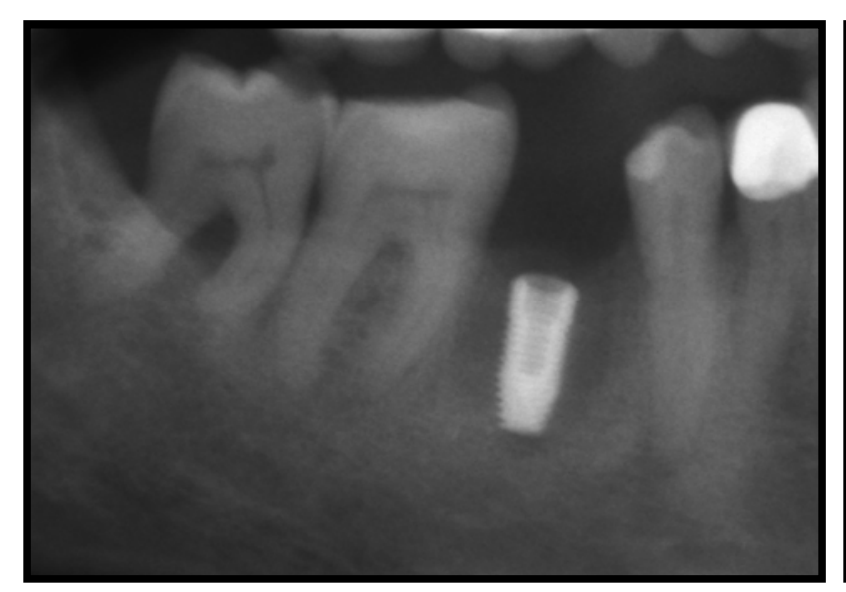

Fig 5: X-ray after insertion

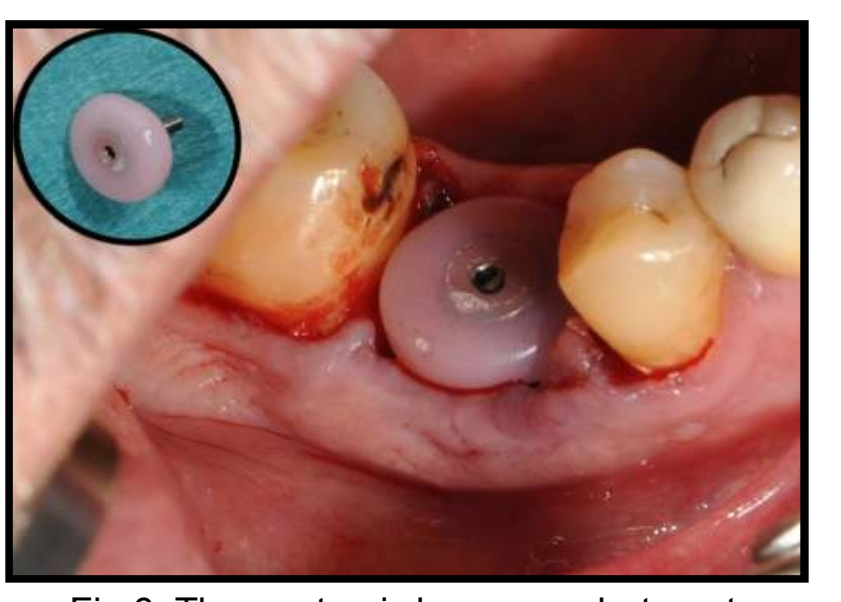

Fig 6: The anatamic harmony abutment

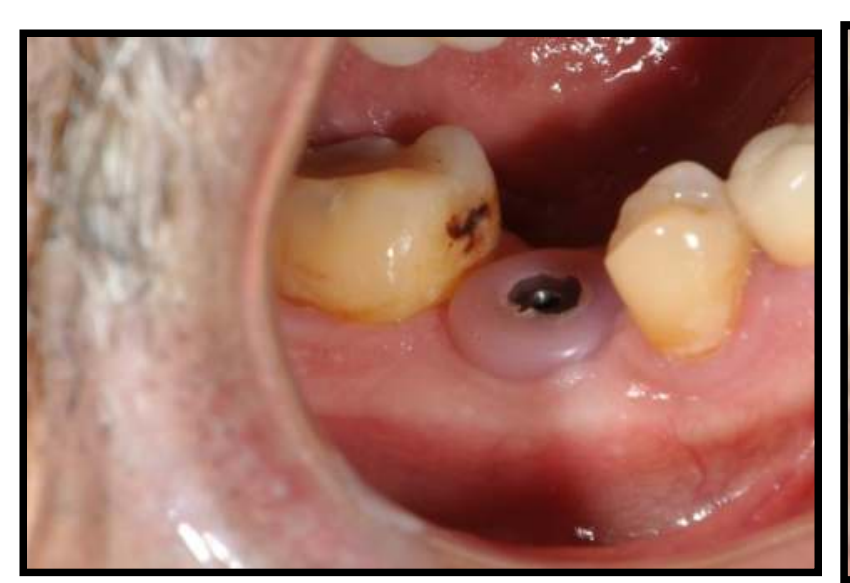

Fig 7: After 2 weeks

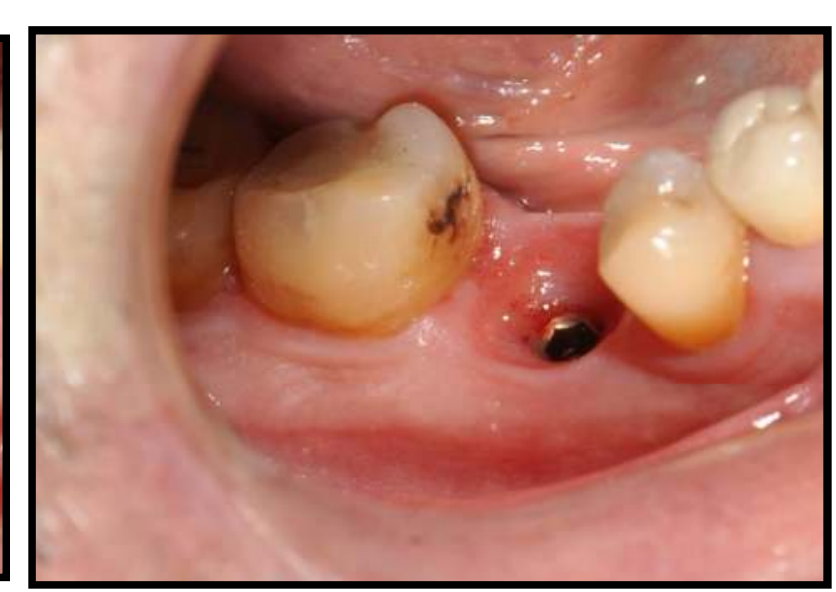

Fig 8: Periimplant mucosa
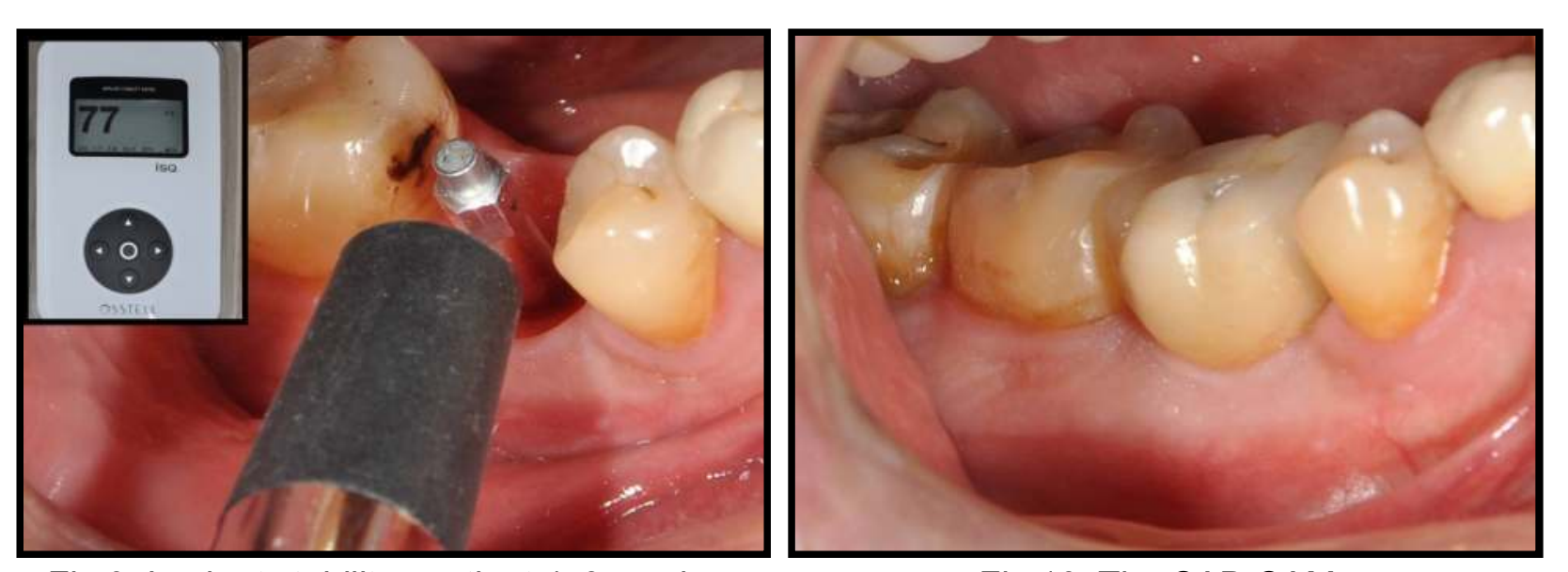

Fig 10: The CAD CAM screw retained restoration

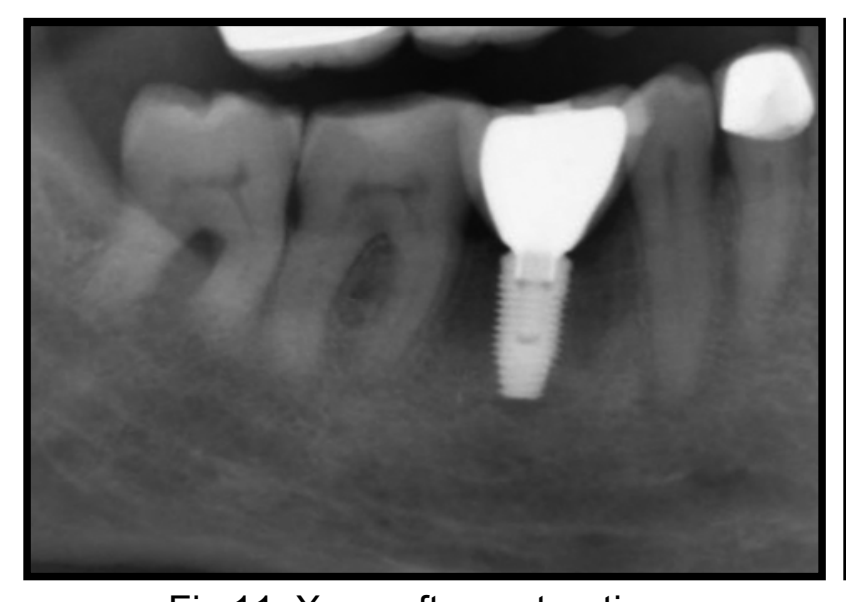

Fig 11: X-ray after restoration

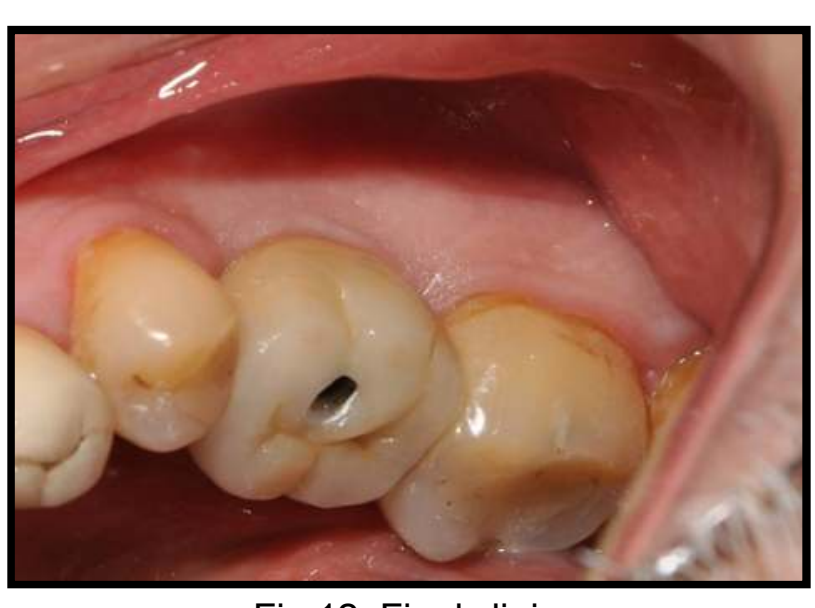

Fig 12: Final clinic

\section{References}

1- Akin R. A New Concept in Maintaining the Emergence Profile in Immediate Posterior Implant Placement: The Anatomic Harmony Abutment. J Oral Maxillofac Surg. 2016 Dec;74(12):23852392

2- Smith RB, Tarnow DP, Sarnachiaro G. Immediate Placement of Dental Implants in Molar Extraction Sockets: An 11-Year Retrospective Analysis. Compend Contin Educ Dent. 2019 Mar;40(3):166-170.

3- Stumpel LJ, Wadhwani C. A Customized Healing Abutment for Immediate and Delayed Implant Cases. Compend Contin Educ Dent. 2017 Nov/Dec;38(10):672-678. 